\title{
Research on Suitable Matching Area in Geomagnetic Navigation
}

\author{
Jing XIAO ${ }^{1, \text { a }}$, Xiusheng DUAN ${ }^{1, b^{*}}$, Xiaohui $\mathrm{Q}^{1, \mathrm{c}}$ and Jiwei ZHONG ${ }^{2}$ \\ ${ }^{1}$ Department of UAV Engineering, Ordnance Engineering College, Shijiazhuang, 050003, China \\ ${ }^{2}$ Department of Electronic Engineering, Special Warfare School, Guangzhou, 510000, China \\ aemail:xiao_jing801@163.com, bemail:sjzdxsh@163.com, cemail:qi-xh@163.com
}

Keywords: Geomagnetic Navigation, Geomagnetic Model, Suitable Matching Area, Feature Extraction

\begin{abstract}
In geomagnetic navigation, the precision and matching efficiency are highly depended on performance of matching area's suitability. The paper summarizes the status and shortages of some main problems in choosing a suitable area, including feature extraction, building of matching feature system, analyzing and predicting of matching suitability and valuing of the matching suitability. It points out several problems that need to be taken into consideration when choosing a matching area, which helps to put forward and optimize the area suitability choosing strategy.
\end{abstract}

\section{Introduction}

The geomagnetic navigation is a way that based on geographical information. It has the characteristics of passive, covert and nonradiative. Above all, the navigation error does not accumulate with time. As a result, it can be used in all-terrain navigation at any time, especially in cross-sea navigation. However, because of the accuracy of current equipment, geomagnetic navigation now is only an aided way and is usually combined with inertial and satellite navigation.

Choosing of matching-suitable area is very important in geomagnetic aided navigation. Matching suitability is the adaptability of matching method to a geomagnetic map, namely the ability that magnetic features describe the geographical position in matching location [1]. Matching-suitable area is the area in magnetic map with excellent matching performance. Navigating in Matching-suitable area will have high precision and matching efficiency. Generally speaking, choosing of matching-suitable area concludes the next parts: building matching feature system, analyzing and predicting matching suitability and valuing matching suitability, among which, the first is the foundation and the second is the key factor.

\section{Confirmation of Navigation Features}

The geomagnetic field is described by seven elements. These are the declination angle $\mathrm{D}$, the inclination $\mathrm{I}$, the northerly intensity $\mathrm{X}$, the easterly intensity $\mathrm{Y}$, the vertical intensity $\mathrm{Z}$, the total intensity $\mathrm{F}$ and the horizontal intensity $\mathrm{T}$. Besides, with the development of different geomagnetic models, for example the main magnetic model, the magnetic anomaly model and the enhanced magnetic model, the navigation feature has a large amount of choice.

In terms of physical significance, navigation feature can be divided into intensity information, angle information and gradient information. And in amount of navigation features, it can be regarded as single factor and multi-feature parameters. If navigation origin is considered, it also can be classified as features based on main magnetic field and anomaly magnetic field. The reason why gradient feature is put forward is to avoid the influence of magnetic field's daily change. Choosing anomaly magnetic field for navigation is because the main magnetic field may not have distinct change at some areas, which may lead to false matching. Table 1 gives origin and accuracy of some reported navigation features [2-6]: 
Table 1 Navigation feature examples.

\begin{tabular}{ccc}
\hline Navigation Features & Origin & Accuracy \\
\hline Anomaly magnetic field & Field measurement & About $200 \mathrm{~m}$ \\
D and I & WMM2010 & $\begin{array}{c}\text { Precision without noise: about } 10 \mathrm{~km} \\
\text { Precision with noise: about } 30 \mathrm{~km}\end{array}$ \\
$\mathrm{~F}$ & IGRF11 & Combined with INS: RMSE $\approx 10$ \\
D and F & WMM2005 & No practical experiment \\
$\mathrm{X}, \mathrm{Y}, \mathrm{Z}$ & IGRF11 & No practical experiment \\
Magnetic field gradient & Calculated by magnetic & Simulation precision less than $100 \mathrm{~m}$ \\
\hline
\end{tabular}

In fact, choosing proper features for navigation is a complicated problem, which considers not only the performance of magnetic sensor, the information involved in the feature, the fluctuation of the geomagnetic field, but also the influence of time-varying field. It is stated that navigation features should take the next factors synthetically [7]:

(1) The feature should be steady in a long term;

(2) The feature should not be influenced by short-term magnetic field change;

(3) The feature should be closely related to the geographical location;

(4) The measurement equipment of the feature should meet the requirements of navigation;

(5) The information of the feature should meet the demands of matching method.

Currently, navigation feature selection is mostly based on experience or qualitative analysis, lacking of quantitative analysis.

\section{Build Basic Matching-suitable Feature System}

Basic matching-suitable features are the features detected from geomagnetic map. They are used for evaluating the matching suitability of an area. At present, building of basic matching-suitable feature system usually refers to related theories in terrain matching. From different point of view, basic matching-suitable feature can be divided into different types. Here is one classification according to its function:

(1) Features that reflect the fluctuation of the geomagnetic field. For example, the mean value of the magnetic field, mean value of accumulation gradient, standard deviation of grade and so on. These features describe the magnetic field respectively both at the macroscopic and microcosmic level.

(2) Features that reflect the information of the geomagnetic field. For example, the Fisher information and the entropy of the magnetic field, which measured the abundance of magnetic field in a certain area.

(3) Features that reflect the uniqueness of the geomagnetic field. For example, the coefficients of association and the coding distortion of the geomagnetic field.

Table 2 lays out several familiar basic matching-suitable features [8-13].

Table 2 Familiar basic matching-suitable features.

\begin{tabular}{lll}
\hline Matching-suitable Features & \multicolumn{1}{c}{ Macroscopic feature } & \multicolumn{1}{c}{ Microcosmic feature } \\
\hline \multirow{2}{*}{ Fluctuation of magnetic field } & $\begin{array}{l}\text { mean value, standard deviation, } \\
\text { accumulation gradient mean } \\
\text { value, coefficient of kurtosis, } \\
\text { coefficient of skew }\end{array}$ & $\begin{array}{l}\text { roughness, ratio of roughness and } \\
\text { standard deviation, standard } \\
\text { deviation of grade, fractal } \\
\text { dimension } \\
\text { Information of magnetic field }\end{array}$ \\
Fisher information & entropy \\
Uniqueness of magnetic field & coding distortion, coefficient of association \\
\hline
\end{tabular}


With the development and introduction of new concepts in information theory and statistics, the matching-suitable feature continues to be perfected.

\section{Methods for Analyzing Matching-suitable Area}

Zitova put forward the matching suitability problem in vision matching [14], Pang S. N. analyzed mechanism of false matching and built a prediction model for image matching [15]. Goldenberg pointed out usability of the magnetic field is closely related to the suitability of a selected area [16]. Synthetically, methods for analyzing matching area can be understood from the next aspects:

(1) Decision-making with multi-features.

The main idea of the method is taking matching-suitable features as the basic properties of decision-making, and with proper method, a new synthetic feature is constructed, by which the candidate matching areas are sorted in order. As a result, the area with the best synthetic feature is the matching-suitable area. This method guarantees the consistency between the synthetic feature and the suitability index.

There are many methods to construct the synthetic feature. In [17], it is calculated by sum operation with coefficients of basic matching-suitable features, but the method mainly depends on human factor and hard to control. In order to avoid this problem, some new methods such as principle component analysis (PCA) [18] and analytic hierarchy progress (AHP) [19] are introduced to the decision making scheme. Results show these methods usually have good performance. Wang Peng constructed the evolutional synthetic feature based on genetic expression programming [20]. By selecting the basic matching-suitable features adaptively, their inner advantages are mined sufficiently.

(2) Decision-making by model prediction.

The main idea of the method is to build the accurate model between basic matching-suitable features and the suitability index to predict suitability of candidate areas. Familiar modeling methods are curve (or hook face) fitting and expression establishing [21] [22].

(3) Decision-making by pattern classification.

The main idea of the method is on basis of the matching-suitable features, the candidate matching areas are sorted into suitable and unsuitable matching pattern by some sorting techniques. The sorting techniques can be discriminated analysis [22], classifier method and hierarchy screening method [23] and so on. Reference [20] generated an adaptively optimistic classifier to select the matching-suitable area, using a GA-SVM algorithm.

Among the three decision-making methods mentioned above, the first two analyze the matching suitability of a certain area qualitatively, where the last gives conclusion quantitatively. Comparatively, decision-making based on multi-features introduces human factor and makes the process subjective. On the other hand, the relationship between the basic matching-suitable features and the suitability index is too complex to accurately model. On the contrary, decision-making based by pattern classification can reduce human factor at the greatest extent. What's more, the complex relationship is integrated into the classifier, avoiding modeling process. It is considered the most important in future matching suitability study.

\section{Matching Suitability Evaluating}

Many indexes are put forward to evaluate the matching suitability, such as the matching probability, matching precision, matching error, capture probability and the circular probable error. In them, matching suitability is widely used. But at present, how to calculate it does not have a uniform standard. In [24], a computing method based on experiment statistic is defined:

$$
P_{C M A}=\frac{\sum_{p \in C M A} \operatorname{CMP}(p)}{N_{C M A}}
$$


In expression (1), CMP (p) represents the right times when a pattern centered on point $\mathrm{p}$ matches in candidate matching areas. When CMP $(p)$ is 1 , it means the matching is right and when it is 0 , the matching fails. $C M A$ represents the candidate matching area, $\sum_{p \in C M A} \operatorname{CMP}(p)$ is the total number of right
matching, and $N_{C M A}$ is the number of experiment.

In general decision-making scheme, matching suitability evaluating acts only a way to determine whether the suitability analysis and prediction is reasonable. In fact, result of suitability evaluating can feed back to the analysis and prediction process, which is helpful to direct the matching-suitable area selection. Fig. 1 is an improved flow chart for matching-suitable area selection.

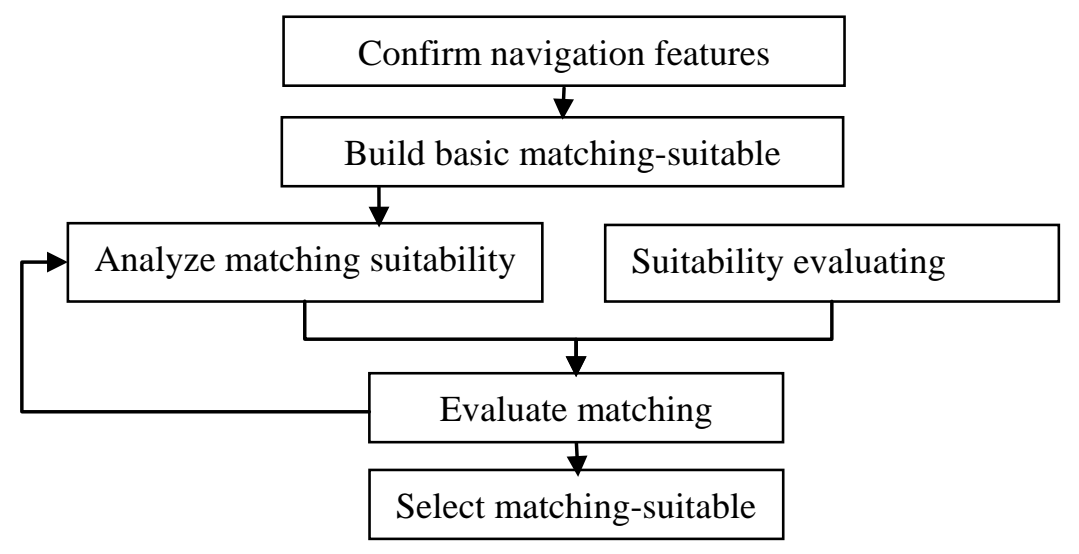

Fig.1. Flow chart for selecting matching-suitable area in geomagnetic navigation.

\section{Summary}

Based on the analysis above, the next conclusions are gotten:

(1) Confirming navigation feature is precondition of the matching-suitable area selection. Only on basis of proper navigation feature can we build the basic matching-suitable feature system specifically and direct matching-suitable area selection.

(2) Decision-making based by pattern classification can reduce human factor at the greatest extent. What's more, the complex relationship is integrated into the classifier, avoiding the modeling process.

(3) Process of evaluating matching suitability verifies the prediction of suitability. Meanwhile, the result can be used to direct the optimization of decision-making scheme.

\section{References}

[1] Shen Lincheng, Bu Yanlong, Xu Xin. Research on Matching-area Suitability for Scene Matching Aided Navigation[J]. Acta Aeronautica Et Astronautica Sinica, 31 (2010) 553-563.

[2] Chen Longwei. Research on Spatial Domain Numerical Methods for Potential Field Continuation in Geomagmetic Navigation[D]. National University of Defense Technology, 2013.

[3] Zhao Zhongwei. Principle and Application of Navigation Based on Magnetic Field Information[D]. Zhejiang University, 2014.

[4] Duan Benyin. Research on Geomagnetic Aided Ins Integrated Navigation System[D]. Harbin Institute of Technology. 2014.

[5] Jiang Lingling. Pure Geomagnetic Homing Navigation on Earth Surface[D]. Zhejiang University, 2012.

[6] Chen Xiaozhi. Federal Filtering Algorithm of Heading Angle Based on INS/GPS/Magnetometer Integrated System[D]. Nanchang University, 2012. 
[7] LIU Fanming, ZHANG Yingfa, QIAN Dong. Full Tensor Geomagnetic Gradient Reference Map Building and Its Integrated Navigation Algorithm[J]. Acta Geodaetica et Cartographica Sinica, 3 (2014) 322-328.

[8] QI Wei, WANG Xiufang, LI Xihai. Selection of characteristic components for geomagnetic matching based on statistical modeling[J]. Progress in Geophysics, 1 (2010) 324-330.

[9] LIU Yuxia, ZHOU Jun, GE Zhilei. A Projecting Pursuit-Based Selection Method for Matching Region in Geomagnetism Navigation[J]. Journal of Astronautics, 31 (2010) 2678-2682.

[10]CHEN Yourong, YUAN Jianping. Research on Matching Suitability of Geomagnetism Map Based on Fractal Dimension[J]. Flight Dynamics, 27 (2009) 76-79.

[11]JIAO Wei, LIU Guangbin, ZHANG Jinsheng. Immune PSO Algorithm-Based Geomagnetic Characteristic Area Selection[J]. Journal of Astronautics, 31 (2010) 1547-1551.

[12] SHI Zhiyong, XU Yang. Algorithm of Geomagnetic Matching Localization based on Entropy[J]. Fire Control \& Command Control, 35 (2010) 8-10.

[13]Li Ting, Zhang Jinsheng, Wang Shicheng. Study on Selection of Geomagnetic Suitable Matching Area Based on Improved Geomagnetic Entropy[J]. Journal of Geodesy and Geodynamics, 5 (2014) 151-155.

[14]Zitova B, Flusser J. Image registration methods: a survey[J]. Image and Vision Computing. 21 (2003) 977-1000.

[15]Pang S N, Kim H C, Kim D, et al. Prediction of the suitability for image-matching based on self-similarity of vision contents[J]. Image and Vision Computing. 22 (2004) 355-365.

[16]Goldenberg F. Geomagnetic navigation beyond magnetic compass[C]. PLANS. San Diego. California, 2006: 684-694.

[17]XU Hanling, YANG Jie, DENG Zhipeng. Flight Path Planning Based on the Evaluation Values of Image Matching Quality[J]. Journal of Shanghai Jiaotong University, 35 (2001) 1336-1339.

[18] Wang P, Wu Y X, Hu X P, et al. Geomagnetic aided navigation suitability evaluation based on principal component analysis[C]. 2012 International Conference on Industrial Control and Electronics Engineering, IEEE Computer Society, Xi'an, China, 23-25, August, 2012 324-329.

[19]WANG Zhe, WANG Shicheng, ZHANG Jinsheng. A Matching Suitability Evaluation Method Based on Analytic Hierarchy Process in Geomagnetism Matching Guidance[J]. Journal of Astronautics, 30 (2009) 1871-1878.

[20]Wang P, Hu X P, Wu M P. A hierarchical decision-making scheme for directional matching suitability analysis in geomagnetic aided navigation[J]. Proceedings of the Institution of Mechanical Engineers, Part G: Journal of Aerospace Engineering, 228 (2013) 1815-1830.

[21]DU Jing, Zhang Tianxu. Selection of matching region for scene matching[J]. Infrared and Laser Engineering, 32 (2003) 368-371.

[22]Wang K, Li Y, Rizos C. Research on terrain suitability of iterative closest contour point algorithm for underwater navigation[C]. 22nd International Technical Meeting of the Satellite Division of the Institute of Navigation. 22-25, Sept., 2009, Georgia, USA, 2009866 - 870.

[23]ZHANG Xiaochen, FU Mengyin. Selection method for scene matching area based on information entropy[J]. Systems Engineering and Electronics, 5 (2011) 1089-1093.

[24]LI Jun, YANG Xin, YANG Li. Mumford-Shah Model Based Critical Subset Segmentation from Navigation Reference Images[J]. Acta Automatica Sinica, 30 (2004) 45-56. 